\title{
Prevalence of Wound Dehiscence in Patients Attending Tertiary Care Institute of Gujarat- A Cross Sectional Study
}

\author{
Akhilesh G Sukhlecha ${ }^{1}$ \\ ${ }^{1}$ Associate Professor, Department of Surgery, Gujarat Adani Institute of Medical Science, Bhuj, Kutch, Gujarat.
}

\section{Abstract}

Background: Wound dehiscence is a very serious postoperative complication which is associated with very high morbidity and mortality rates. The aims and objectives of the study were: To find out epidemiology of wound dehiscence and to evaluate various predictors leading to wound dehiscence. Subjects and Methods: The study was conducted in the Department of Surgery, Gujarat Adani Institute of Medical Science, Bhuj, Kutch, Gujarat and included all the patients operated for abdominal pathologies by any incision. A detailed history was taken from all the patients or their relatives where patient was not able to give history or was a minor. Thorough general, systemic and local examination was performed in all the patients included in the study. Results: The age of patients varied from 2 days to 82 years, which included a total of 58 patients. The mean age \pm standard deviation was found $49.14 \pm 16.40$ years. Most patients were in the age group of $61-$ 70 years. The study consisted of 42 males and 17 females. Enteric perforation: 13 patients had enteric perforation as diagnosis. Duodenal perforation: 11 had duodenal perforation as basic pathology. Obstruction was found to be present in 09 of burst abdomens. Conclusion: Prior stabilization with adequate hydration and correcting the electrolyte imbalance if any is must before proceeding for emergency laparotomies, Co-morbidities should be addressed as much they can be before proceeding for surgery. Proper sterilization should be ensured in emergency theatres too.

Keywords: Enteric perforation, Epidemiology, Obstruction, Wound dehiscence.

Corresponding Author: Dr Akhilesh G Sukhlecha, 28 new nirmalsinh wadi, near pramukhswami temple, Bhuj, Kutch, Gujarat, India-370001.

Received: May 2019

Accepted: June 2019

\section{Introduction}

Wounds and their management are fundamental to the practice of surgery, as any surgical intervention will result in a wound. Postoperative wound complications represent one of the most frustrating and difficult occurrences experienced by surgeons. Wound dehiscence is a very serious postoperative complication which is associated with very high morbidity and mortality rates. It affects the patients by increasing distress and risk of mortality, the attendants by increasing the cost of treatment and wastage of their precious time in hospital, the surgeon for whom it is a disturbing reality and the hospital resources by increasing the healthcare cost due to prolonged stay. Abdominal wound dehiscence occurs in $3.6 \%$ to $29 \%$ of all obstetrics and gynecology laparotomies, ${ }^{[1,2]}$ and frequently presents with prolonged wound healing and continued drainage. Multiple host-related factors, including obesity, smoking, surgical site infection, which occurs in roughly $3 \%$ to $20 \%$ of cesarean births, ${ }^{[3,4]}$ have been shown to inhibit wound healing. Other technical factors, such as inadequate hemostasis leading to the accumulation of seroma or hematoma, can also impede wound healing. Similarly, post-operative increases in intraabdominal pressure due to ileus, vomiting, or coughing can place tension on the wound, and thus increase the probability of separation. ${ }^{[5]}$
Empirically, the ancients recognized that foreign bodies and dead tissues must be removed from wounds to prevent wound complications. ${ }^{[6]}$ Joseph Lister in 1865 was the first to use carbolic acid to prevent wound dehiscence. However, fellow surgeons advocated proper hand wash with antiseptic, sterile clothes, sterile linen. Surgical instruments were sterilized with autoclave. Ehrlich, Flemming and Florey realized, with increasing sophistication, that bacteria were pathogens that prevent healing and led to sepsis and death. Control of bacteria by asepsis, antiseptics and antimicrobials heralded a new era in wound management. ${ }^{[7]}$

Wound dehiscence is an acute wound failure. The rate of wound dehiscence reported in international literature varies from to 1 to $2.6 \%$, while Indian subcontinent study shows and incidence much higher, as high as 6 to $12 \%$, which is unacceptable and alarming. ${ }^{[8-10]}$ The associated mortality with wound dehiscence has been described within the range of 15 to $50 \%$. $^{[11]}$

\section{The aims and objectives of the study were:}

1. To find out epidemiology of wound dehiscence.

2. To evaluate various predictors leading to wound dehiscence.

3. Based on the observations, measures will be recommended for prevention of abdominal wound dehiscence. 


\section{Subjects and Methods}

The study was conducted in the Department of Surgery, Gujarat Adani Institute of Medical Science, Bhuj, Kutch, Gujarat and included all the patients operated for abdominal pathologies by any incision. The study included patients irrespective of age, sex, region, religion, profession, disease, type of surgery, duration of surgery and type of anaesthesia.

Patients undergoing laparoscopic, minimal invasive urological/ gynaecological, vascular, thoracic-abdominal procedures and herniorraphies were not included, so were the patients who did not give their consent for the study. All the patients and/or their relatives were explained in detail about the study. A written consent was taken from all the patients or their relatives. A detailed history was taken from all the patients or their relatives where patient was not able to give history or was a minor. Thorough general, systemic and local examination was performed in all the patients included in the study.

\section{Results}

The present study comprised 500 patients operated for abdominal pathologies by any incision. The mean age of patients was around 36 years. There were 382 males and 118 females who were operated upon during one year period. Examination of wound was done after 24 hours. Postoperative duration of swelling, discharge, dehiscence was recorded in detail, all other postoperative complications or aggravation of already existing diseases was also recorded. The study observed patients with wound dehiscence. The age of patients varied from 2 days to 82 years, which included a total of 58 patients. The mean age \pm standard deviation was found $49.14 \pm 16.40$ years. Most patients were in the age group of $61-70$ years. [Table 1] The study consisted of 42 males and 17 females. Enteric perforation: 13 patients had enteric perforation as diagnosis. Duodenal perforation: 11 had duodenal perforation as basic pathology. Obstruction was found to be present in 09 of burst abdomens.

\section{Table 1: Age incidence of patients with diabetic mellitus}

\begin{tabular}{|l|l|l|}
\hline Sr. No. & Age Group & Number \\
\hline 1 & $40-50$ years & 50 \\
\hline 2 & $51-60$ years & 50 \\
\hline 3 & Total & 100 \\
\hline
\end{tabular}

\section{Table 2: Distribution of types of Presentation}

\begin{tabular}{|l|l|l|}
\hline Sr. No. & Types of Presentation & No. of cases \\
\hline 1 & Ulcer & 75 \\
\hline 2 & Cellulites & 15 \\
\hline 3 & Gangrene & 15 \\
\hline 4 & Total & 100 \\
\hline
\end{tabular}

Table 3: Common bacteria isolated those with non-gangrenous characteristics.

\begin{tabular}{|l|l|l|}
\hline Sr. No. & Name of Organism & Percentage of cases \\
\hline 1 & Staphylococcus aureus & $55 \%$ \\
\hline 2 & Gram Negative Organisms & $25 \%$ \\
\hline 3 & Beta Haemolytic Streptococci & $8 \%$ \\
\hline 4 & Anaerobic Cocci & $10 \%$ \\
\hline
\end{tabular}

Table 4: Types of operations performed $(n=100)$

\begin{tabular}{|l|l|}
\hline Type of operation & Frequency \\
\hline Debridement & 25 \\
\hline Amputation & 60 \\
\hline Skin Grafting & 5 \\
\hline Incision \& Drainage & 4 \\
\hline Sequestrectomy & 6 \\
\hline
\end{tabular}

Table 5: Wagner's classification of diabetic foot ulcers

\begin{tabular}{|l|l|l|}
\hline Sr. No. & Ulcer Grading & Description \\
\hline 1 & Grade 0 & No ulcer but high risk foot \\
\hline 2 & Grade 1 & Superficial ulcer \\
\hline 3 & Grade 2 & $\begin{array}{l}\text { Deep ulcer, no bony involvement or } \\
\text { abscess }\end{array}$ \\
\hline 4 & Grade 3 & Abscess with bony involvement \\
\hline 5 & Grade 4 & Localized gangrene eg. toe, heel, etc \\
\hline 6 & Grade 5 & $\begin{array}{l}\text { Extensive gangrene involving the whole } \\
\text { foot }\end{array}$ \\
\hline
\end{tabular}

\section{Discussion}

Both patient-related factors and non-patient factors contribute to surgical wound dehiscence after abdominal laparotomy. Unfortunately, there is wide variability in which patient and non-patient factors have been included in the various studies, examining laparotomy dehiscence. A retrospective review of a patient registry conducted by van Ramshorst et al. compared 363 all-cause cases of abdominal wound dehiscence to matched controls. They found that the major independent risk factors predictive of abdominal wound dehiscence included age, gender, chronic pulmonary disease, ascites, jaundice, anemia, and emergency surgery, type of surgery, postoperative coughing, and wound infection. ${ }^{[12]}$

The results of our study are comparable to studies done in Indian sub-continent. In our study 59 out of 500 patients who underwent surgeries in Department of General Surgery during one year period developed wound dehiscence giving an incidence of $1.79 \%$, which is much higher as compared to developed countries but comparable to Indian sub-continent. Similar results were observed by Amin AQ et al (2013) in which 15 out of 130 developed wound dehiscence $(11.5 \%)$. Numerous reasons have been ascribed for such higher incidence. ${ }^{[13]}$ In our study the high rate of wound dehiscence is due to many reasons. In our set up the emergency surgeries are usually performed for acute abdomen cases which have been deteriorated due to course of acute illness as patients are from far flung areas. In our part of world peripheral hospital set up is not up to mark. Diagnostic studies are not available due to which patient who needs surgery are managed conservatively there are referred to higher centre when their condition deteriorates. Many patients are mismanaged by Aalims, quacks, hakims and local dispensers. Most of the patients already have complications like septicemia, fluid and electrolyte derangements at time of presentation to tertiary centre. Also, poverty plays a vital role in making patients malnourished and compelling them to seek cheaper treatment outside hospitals at local dispensaries.

The age of patients in current study ranged from 2 days to 80 years with mean age of 49 years. The highest incidence in present series is between $61-70$ years. According to AIShamarry SAR (2002) mean age is 58 years and affected 
group was 61 to 70 years. ${ }^{[13]}$ The observation coincides with Hampton's observation which considers that there is a co relation but not a cause and effect relationship. One of the interesting risk factor found in our study, is gender. In previous studies, males have been reported to have higher risk of developing abdominal wound dehiscence. The reason of this disadvantage is not entirely clear, one of the possible confounders may be smoking. Because most smokers from the studied generation tended to be male, and the effect of gender may be confounded with effect of smoking on wound healing. Smokers tend to have cough and more chances of acid peptic disease. Another explanation may be that men tend to build higher abdominal pressure than females. An increase in intra-abdominal pressure results in higher strain on the wound edges. In our study 42 of patients were male and remaining 17 were females. According to Wergar SH (2005) percentage of male were $11 \%$ and female were $29 \%$. In the present study it was noted that pre-operative predisposing factors like anaemia, chronic obstructive pulmonary disease, sepsis, diabetes, jaundice etc. were associated with increased incidence of burst abdomen. In many patients, there was more than one factor leading to occurrence of burst abdomen. Joergenson and Smith noticed in their study.

In our study the high rate of wound dehiscence is due to many reasons. In our setup the emergency surgeries are usually performed for acute abdomen cases which have been deteriorated due to course of acute illness as patients are from far flung areas. In our part of world peripheral hospital set up is not to mark, diagnostic studies are not available due to which patients who need surgery are managed conservatively there and referred to higher centre only when their condition deteriorates. Most of the patients are already having complications like septicemia and fluid and electrolyte derangements at the time of presentation to tertiary hospital. Also, poverty plays a vital role in making patients malnourished and compelling them to seek cheaper treatment outside hospital at local dispensaries. Second factor responsible in emergency cases may be lack of proper sterilization in emergency setup. Third factor which plays a vital role in developing wound dehiscence is lack of experience on part of surgeon as emergency laparotomies are performed most of the time by surgical residents. Fourth factor is, since patients are low and most of the time closure is done in hurry without giving further muscle relaxant by anesthetist.

\section{Conclusion}

Prior stabilization with adequate hydration and correcting the electrolyte imbalance if any is must before proceeding for emergency laparotomies, Co-morbidities should be addressed as much they can be before proceeding for surgery.Proper sterilization should be ensured in emergency theatres too.

\section{References}

1. Ferres MA, Olivarez SA, Trinh V, Davidson C, Sangi-Haghpeykar H, Aagaard-Tillery KM. Rate of wound complications with enoxaparin use among women at high risk for postpartum thrombosis. Obstet Gynecol. 2011;117(1):119-24

2. Pitkin RM. Abdominal hysterectomy in obese women. Surg gynecol obstet. 1976;142(4):532-6.

3. Schneid-Kofman N, Sheiner E, Levy A, Holcberg G. Risk factors for wound infection following cesarean deliveries. Int $\mathrm{J}$ Gynaecol Obstet. 2005;90(10:10-15.

4. Kajiwara K, Eizo Kimur, Makoto Nakano, Hirokuni Takano, Aikou Okamoto. Clinical experience of J- VAC drains for skin closure in the laparotomy of obstetrics and gynecology. J Obstet Gynaecol Research. 2014;40(40):1089-97.

5. Helmkamp FB. Abdominal wound dehiscence. Am J Obstet Gynecol. 1977;128 (7):803-7.

6. Agrawal V, Sharma N, Joshi MK, et al. Role of suture material and technique of closure in wound outcome following laparotomy for peritonitis. Tropical Gastroenterology 2009; 30(4): 237-240.

7. Cavit COL, Atilla $\mathrm{S}$ and Melten COL. Can postoperative Abdominal Wound Dehiscence be predicated? Tokai Journal Exp Clinical Medicine, 1998; 23(3): 123-127.

8. Efron G. Abdominal wound disruption. Lancet 1965; 1: 1287-1290.

9. Fleischer GM, Rennert A and Ruhmer M. Infected abdominal wall and burst abdomen. Chirurg 2000; 71: 754-762.

10. Granam DJ, Stevenson JT and Mettenry CR. Association of intraabdominal infections and abdominal wound dehiscence. American Surgery 1998; 64(7): 660-665.

11. Sarsam SE, Elliott JP, Lam GK. Management of wound complications from cesarean delivery. Obstet Gynecol Survey. 2005;60(7):462-73.

12. van Ramshorst GH, Nieuwenhuizen J, Hop WC, Arends P, Boom J, Jeekel $\mathrm{J}$, et al. Abdominal Wound Dehiscence in Adults: Development and Validation of a Risk Model. World J Surg. 2010;34.1:20-27.

13. Amini AQ, Khan NA, Ahmad J, et al. Management of abdominal wound dehiscence: still a challenge. Pakistan Journal of Surgery 2013; 29(2): 84 87.

Copyright: () the author(s), 2019. It is an open-access article distributed under the terms of the Creative Commons Attribution License (CC BY 4.0), which permits authors to retain ownership of the copyright for their content, and allow anyone to download, reuse, reprint, modify, distribute and/or copy the content as long as the original authors and source are cited.

How to cite this article: Sukhlecha AG. Prevalence of Wound Dehiscence in Patients Attending Tertiary Care Institute of Gujarat- A Cross Sectional Study. Acad. J Surg. 2019;2(1):7-9.

DOI: dx.doi.org/10.21276/ajs.2019.2.1.3

Source of Support: Nil, Conflict of Interest: None declared. 